International Review of Community Development

Revue internationale d'action communautaire

\title{
L'autonomie des collectivités locales : entre la crise et le changement
}

Local autonomy: Crisis and change

La autonomía de las colectividades locales: entre la crisis y el cambio

\section{Annick Germain et Pierre Hamel}

Numéro 13 (53), printemps 1985

Les ambiguïtés du pouvoir local

URI : https://id.erudit.org/iderudit/1034535ar

DOI : https://doi.org/10.7202/1034535ar

Aller au sommaire du numéro

Éditeur(s)

Lien social et Politiques

ISSN

0707-9699 (imprimé)

2369-6400 (numérique)

Découvrir la revue

Citer cet article

Germain, A. \& Hamel, P. (1985). L'autonomie des collectivités locales : entre la crise et le changement. International Review of Community Development / Revue internationale d'action communautaire, (13), 35-45.

https://doi.org/10.7202/1034535ar
Résumé de l'article

Comment poser aujourd'hui la question du pouvoir local ? Ne nous renvoie-t-elle qu'aux débats sur la décentralisation ? Et, au Québec, la décentralisation ne se pose-t-elle pas essentiellement en termes d'aménagement ? L'article de A. Germain et P. Hamel vise à montrer que les enjeux du pouvoir local doivent être saisis à l'intérieur d'une perspective élargie qui ne dissocie pas la réforme des institutions locales de l'évolution des collectivités locales face aux restructurations du développement économique, et des forces sociales diverses qui composent aujourd'hui le paysage de la scène locale. Après avoir montré l'évolution de la signification des réformes gouvernementales selon leur inscription dans des conjonctures très différentes (la période d'expansion du début des années soixante, la crise de la fin des années soixante-dix), les auteurs montrent que ce faisant, les rapports entre État et municipalités ont évolué dans le sens d'une affirmation plus dynamique de ces dernières. Ce dynamisme est aussi lié à la crise, aux restructurations de l'économie qui l'accompagnent. Parties prenantes dans l'aménagement des effets de ces restructurations sur leur territoire, les collectivités locales ont, à la fois, joué un rôle supplétif pour amortir les effets de la crise et pris des initiatives, notamment dans la relance de l'emploi. C'est dire que la voie est étroite entre une évolution dans le sens de la dualisation sociale d'une part, et l'amorce d'un développement plus autonome et autocentré d'autre part. À cet égard, les forces en présence au niveau local pèseront de façon décisive sur l'évolution des sociétés locales.
Tous droits réservés @ Lien social et Politiques, 1985
Ce document est protégé par la loi sur le droit d'auteur. L’utilisation des services d'Érudit (y compris la reproduction) est assujettie à sa politique d'utilisation que vous pouvez consulter en ligne. 


\section{L'autonomie des collectivités} locales : entre la crise et le changement

\section{A. Germain \\ P. Hamel}

Le pouvoir local, un nouveau paradigme?

La question du pouvoir local a, pendant longtemps, été identifiée aux obstacles rencontrés par les politiques de décentralisation, du côté des décideurs, et à la découverte du fonctionnement des sociétés locales dans une veine quasi ethnographique, du côté des chercheurs. Ces deux préoccupations qui sont certes encore très présentes aujourd'hui, s'inscrivent de plus en plus dans des perspectives très différentes, qui font du pouvoir local, non plus un objet particulier, mais une manière de regarder et d'interroger les problèmes de l'heure.

Ce que l'on voudrait montrer ici, c'est que le pouvoir local peut être vu - et l'est d'ailleurs de plus en plus - comme une manière particulièrement intéressante d'analyser les enjeux qui sont au coeur de la crise que traversent nos sociétés, voire comme un moyen d'appréhender les transformations dont elle est porteuse. Celles-ci, telles que nous pouvons les entrevoir, exigent en effet une révision en profondeur des modèles d'analyse et des méthodologies développées depuis, plus particulièrement, les années soixante. L'analyse du social " pur ", pour reprendre le terme de Yves Barel ${ }^{1}$, c'est-à-dire l'analyse des groupes et acteurs saisis au niveau "le plus haut de la société ", celui de la société globale - qu'on la nomme formation sociale ou système d'action historique - est délaissée par de nombreux chercheurs. Non pas qu'il faille substituer une sociologie de l'intégration à une sociologie du conflit, ou qu'il faille choisir entre l'une et l'autre. Ce que nous apprend l'analyse du pouvoir local, c'est la richesse d'une perspective ne dissociant pas l'étude du micro-social de celle du macrosocial, une perspective maniant le paradoxe, c'est-à-dire travaillant sur deux niveaux à la fois, sans les polariser par une dichotomie des contraires.

Effet de connaissance et effet de conjoncture relèvent donc d'un rapport de réciprocité : la crise structurelle que traversent nos sociétés contribue à déstabiliser nos schémas d'analyse au moment où ces derniers font l'objet de remises en question internes et externes. Quelles sont donc les questions nouvelles que pose, aujourd'hui, le pouvoir local ? À quels types de phénomènes faut-il rattacher l'intérêt renouvelé pour le local? Dans quelle mesure le local devient-il un lieu stratégique de recomposition sociale dans le contexte de crise ? N'y a-t-il pas dorénavant élabora- 
tion de nouvelles perspectives de développement qui se définissent précisément à partir du local ? Et est-ce que ceci ne coïncide pas avec la production d'un nouveau consensus au sujet du rôle de l'État qui se construirait principalement à partir de ce niveau? Ce qui signifierait que non seulement les rapports entre l'État et la société civile mais aussi les rapports qu'entretiennent entre eux et avec l'État les principaux acteurs de la société civile se transforment profondément. Et c'est sans doute en ce sens qu'il faut interpréter les débats réouverts récemment tant par le néocorporatisme que par le néolibéralisme concernant le rôle de l'État comme principal agent de négociation et de redistribution sociale.

Notre intention ici est de mettre en perspective, en référence au cas québécois, les principaux éléments du débat relatifs aux enjeux du pouvoir local. C'est pourquoi, dans un premier temps, il nous a semblé nécessaire de partir des processus de redéploiement industriel et de " mutation des économies locales ". Ceux-ci sont à l'origine des stratégies économiques qui se répercutent sur les rapports de force entre les acteurs sociaux qui interviennent sur la scène locale. Et, jusqu'à un certain point, ils nous semblent déterminants à l'égard des bouleversements déjà invoqués.

Mais les stratégies économiques ne sont pas seules en cause. La scène locale est aussi le lieu privilégié de destination des réformes politico-administratives des dernières années. À ce titre elle est l'objet d'une attention particulière de la part des paliers supérieurs de gouvernement qui tentent de s'ajuster à une nouvelle réalité sociale et économique. Cependant, fait récent, les pouvoirs locaux ne réagissent plus de façon passive aux projets gouvernementaux. Leur dynamisme les amène à prendre des initiatives et, dans certains cas, à revendiquer davantage d'autonomie. C'est en quelque sorte ces réajustements institutionnels qu'il convient de prendre en compte dans un deuxième temps.

Enfin, si certaines transformations font déjà partie des nouvelles règles du jeu, d'autres sont toujours à venir. Les rapports de force entre les acteurs de la société civile ne sont pas immuables. L'évolution de la conjoncture, l'émergence de nouveaux enjeux peuvent faire basculer des positions qui apparaissaient relativement stables auparavant. Dans ce contexte il nous semble que les mouvements sociaux risquent d'être menacés par le rééquilibrage des forces sur la scène locale, surtout s'ils ignorent le sens des transformations en cours. C'est ce que nous examinerons en dernier lieu.

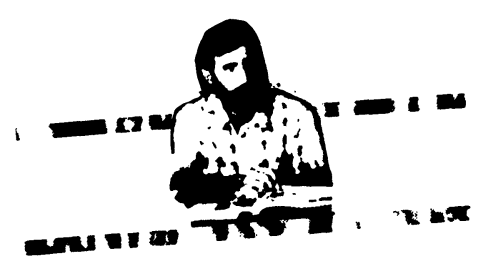

\section{Les collectivités locales et la crise}

Par collectivités locales nous désignerons ici l'ensemble des groupes qui participent à la gestion du pouvoir sur un territoire donné. Ainsi pour nous la collectivité locale n'est pas synonyme de municipalité même si son action peut se déployer à l'intérieur du même périmètre territorial. Cette mise au point étant faite, soulignons, d'entrée de jeu, que parmi les éléments qui caractérisent les transformations sociales des dernières années, il faut mentionner le changement d'attitude des collectivités locales à l'égard du développement. C'est ce que traduisent, entre autres, les initiatives émanant des milieux locaux et qui concernent, par exemple, l'innovation industrielle, l'amélioration du cadre de vie ou encore la mise en place de réseaux efficaces de communication et de concertation entre les acteurs socio-économiques en vue de soutenir le développement. De plus l'on constate que, dorénavant, les collectivités locales sont partie prenante des débats et des choix préalables aux reconversions industrielles comme elles se préoccupent davantage des conditions de création d'emplois.

Ces phénomènes possèdent une visibilité qui s'exprime autant à travers une vitalité de l'entrepreneurship local - que confirme notamment le fort taux de création de PME ces dernières années au Québec ${ }^{2}$ - que par le biais d'une volonté collective d'accroître l'autonomie des instances décentralisées. De la sorte on en vient à parler d'une configuration particulière pour cerner la forme que prennent en ce moment les dynamismes locaux et régionaux. C'est ce que propose d'éclairer tout un courant de recherche à l'intérieur de la " science régionale " qui, en mettant de côté les catégories d'analyse formalisées dans le cadre d'une conception mécanique du développement, 
explorent le contenu et les formes actuelles des dynamismes régionaux. Leur problématique est dès lors ouverte sur la complexité des processus multidimensionnels, tant qualitatifs que quantitatifs, inscrits dans les stratégies de développement.

Soulignons que ces chercheurs se démarquent autant des analyses qui soutenaient que la croissance allait permettre d'atténuer les disparités régionales - thèse de la convergence des disparités que de celles qui soutenaient au contraire que les disparités iraient en s'accentuant - thèse du renforcement des disparités. La thèse de la convergence est mise de l'avant d'une part par les néoclassiques qui invoquent l'existence de mécanismes " naturels " d'ajustement et, d'autre part, par les tenants de la polarisation qui soutiennent que la croissance doit se diffuser du pôle vers la périphérie. La thèse du renforcement des disparités a été mise de l'avant par des chercheurs marxistes qui ont montré que le développement du capitalisme a engendré de manière intrinsèque des inégalités territoriales ${ }^{3}$.

Pour comprendre les "nouveaux " dynamismes régionaux il apparaît donc nécessaire de mettre de côté les anciens modèles d'analyse. C'est seulement à cette condition qu'il est possible d'appréhender la dynamique spatiale qui se déroule depuis quelques années et que les catégories d'analyse néoclassiques ou marxistes ne permettent pas bien de saisir. En ce sens Philippe Aydalot identifie un phénomène de " retournement spatial ". II désigne par là un « renversement de tendances " autant sur le plan démographique que sur le plan des dynamismes économiques ${ }^{4}$.

En effet, depuis une dizaine d'années, on observe en France et c'est également le cas aux ÉtatsUnis - des transformations radicales dans la répartition de l'emploi, des activités économiques et de la production dans l'espace. C'est du moins ce que révèle l'analyse de certains indicateurs tels que les migrations inter-régionales, l'évolution des effectifs industriels régionaux ou encore les taux de variation de l'emploi. Or ces manifestations renvoient à des réalités, à des " forces " qui n'agissaient pas auparavant. Pour Philippe Aydalot, " l'obsolescence des activités anciennes et des sites industriels qui les accueillaient ${ }^{5}$ " ou encore "l'incapacité des vieilles régions à se reconvertir ${ }^{6}$ ", sont liées au poids exercé sur ces espaces économiques par des spécialisations qui ne sont plus rentables dans le contexte de la troisième révolution industrielle. Inversement les régions jugées " en retard " dans le cadre du développement polarisé ne sont plus nécessairement pénalisées. Les cartes étant redistribuées, ces régions ont souvent plus de facilité à s'adapter ou à faire valoir des opportunités qui, dans le passé, ne pouvaient être prises en compte mais que, dorénavant, l'introduction des nouvelles technologies favorise. $\mathrm{Ne}$ serait-on pas en train de passer " d'un modèle de développement basé sur la diffusion d'un processus d'origine extérieure, à un modèle internalisé ${ }^{7}$ ", telle est la question que se posent les chercheurs du Centre Économie, Espace, Environnement.

C'est par rapport à ce changement de tendances que les pouvoirs locaux sont plus ou moins directement interpellés. Et c'est à ce niveau qu'il convient, dans un premier temps, de situer les enjeux du local.

L'intervention des acteurs locaux sur le développement ne va pas sans ambiguittés. À quoi correspondent leurs initiatives? $\mathrm{Ne}$ demeurent-elles pas encore fortement marquées par le retrait de l'État et des grandes entreprises qui privilégient les investissements dans les secteurs de pointe? Dans ce cas les collectivités locales ne joueraient-elles pas un rôle supplétif visant essentiellement à amortir les effets de la crise des dernières années? Inversement ne pourrait-on pas considérer, compte tenu des mécanismes de blocage de la croissance inhérents à la crise que ce rôle supplétif demeure somme toute secondaire en comparaison des dynamismes locaux qui s'enracinent dans une volonté collective d'induire un développement endogène? En d'autres termes, même s'il est vrai que les instances politiques rattachées aux paliers centralisés ont tendance à reporter sur les pouvoirs locaux une part grandissante de la gestion des affaires publiques, il semble que les initiatives et les dynamismes locaux évoqués s'enracinent dans une restructuration qui va au-delà de la réorganisation du système de gestion étatique. S'il y a une crise de l'État-providence susceptible d'expliquer - du moins en partie - le réalignement de l'État, notamment à travers un discours critique à l'endroit de la tradition centralisatrice, cette crise ne peut-elle pas à son tour être éclairée par la crise plus profonde qui sous-tend le blocage des mécanismes de croissance. Ne pouvant plus compter exclusivement, comme par le passé, ni sur la grande entreprise ni sur l'État pour soutenir la croissance, les acteurs locaux apprendraient à miser, en premier lieu, sur leurs propres ressources et leurs propres moyens. C'est du moins I'hypothèse qui sous-tend le réajustement du cadre d'action du gouvernement québécois ${ }^{8}$.

Selon que l'on aborde la question sous l'angle du dynamisme des collectivités locales ou sous celui de leur dépendance face aux nouvelles politiques décentralisatrices, l'on s'engage dans deux axes d'interprétation différents.

Sans trancher, pour l'instant, entre les deux, soulignons qu'ils ne sont pas nécessairement mutuellement exclusifs. Ainsi en dépit d'une stratégie étatique en vue d'élargir la gestion de la crise, entre 
38 autres en mettant à contribution les collectivités locales, ces dernières ont pris des initiatives qui débordent le cadre normatif qui leur était proposé. Toutefois la menace d'une forme de "dualisation spatiale sociale " reste toujours présente. En effet les segments dominants de l'État favorisent une restructuration de l'économie prenant appui sur la configuration actuelle du redéploiement industriel dans le cadre du nouvel ordre économique mondial $^{9}$. Dans ce contexte les économies régionales sont appelées à se spécialiser en misant sur les créneaux de pointe qui s'articulent au " virage technologique ". $\mathrm{Ce}$ qui n'empêche pas, dans ce même contexte, les représentants politiques des paliers supérieurs de gouvernement de considérer qu'il est nécessaire de stimuler les initiatives locales, "le communautaire " pour prendre en charge, à moindre coût, les régions ou les secteurs en déclin.

Dès lors nous voilà confrontés à un double discours et à une double pratique dans un environnement en transformation. Les tendances lourdes de l'économie, en quête de nouvelles bases pour l'accumulation du capital, enclenchent progressivement une économie à deux vitesses. À ce sujet le VIle Plan français parlait déjà d'une " croissance couplée ".

Le rapport Nora, qui s'efforce de tracer les contours de la société informatisée de demain, écrit François Dalbert, distingue d'une part " les grandes entreprises vouées à la rationalisation et à la productivité maximum " flanquée de " petites unités performantes ouvrant la voie à l'innovation, aux nouveaux produits, aux nouvelles consommations et dont la loi restera la combativité, le goût du risque et la quête du profit maximum " et, d'autre part, "des services publics, des coopératives, des associations, des groupes très décentralisés, peu soucieux de rendement économique et financier mais consommateurs de main-d'oeuvre et dispensateur d'aménités ${ }^{10}$.

En considérant l'évolution récente du marché de l'emploi on peut se représenter plus concrètement cette économie à deux vitesses qui signifie, pour une couche grandissante de travailleurs et de travailleuses, une précarité accrue - perte de la sécurité d'emploi, entre autres - et une déqualification des tâches d'exécution. Concernant les effets spatiaux de cette dualisation, il n'est pas certain qu'elle accentue de la même manière les inégalités qui s'étaient creusées dans l'économie d'aprèsguerre entre les régions du centre et celles de la périphérie. À la lumière des analyses sur les phénomènes de retournement, nous faisons l'hypothèse que les forces économiques dominantes suscitent une division spatiale qui est modulée par les dynamismes locaux et régionaux alors que les PME, non seulement prennent la relève, comblent les pertes d'emplois, les baisses de production, les diminutions d'effectifs dans les grandes entreprises, mais participent à un développement endogène qui s'inscrit à l'intérieur d'une autre problématique de développement.

Notons toutefois que les tendances à la dualisation spatiale et sociale soutenues par les forces dominantes de l'économie capitaliste et renforcées par certains segments de l'État ne sont pas forcément contrebalancées par les dynamismes locaux et régionaux. Ceuxci peuvent également profiter de... ou accentuer la dualisation. II est bien connu, par exemple, que les
PME et le syndicalisme font rarement bon ménage. De plus les travailleurs et travailleuses de PME sont peut-être portés - question de survie - à accepter plus facilement que les travailleurs et travailleuses des grandes entreprises, des conditions de travail difficiles et des salaires moindres.

Ces remarques qui, à un certain niveau, peuvent sembler contradictoires par rapport à l'existence des « retournements de tendances ", ne les nient pourtant pas. II faut se demander ici à qui profite vraiment l'émergence de dynamismes locaux et régionaux. Est-ce que ceux-ci ne correspondent pas, avant tout, à la montée des bourgeoisies régionales? Les travailleurs et travailleuses, dans cette perspective, ne parviendraient pas à accroître leur contrôle sur le système économique. Sans parler des exclus du système de production, dont certains continueraient à remplir, malgré eux, la fonction d'armée de réserve.

Pourtant dans plusieurs régions du Québec, depuis quelques années, les syndicats participent plus volontiers à des projets de relance économique. Ce faisant ils sont amenés à revoir leur mode d'action traditionnel. À ceci s'ajoutent d'autres formes d'intervention qui se situent à une plus grande échelle comme le Fonds de solidarité de la Fédération des travailleurs du Québec (FTQ) qui vise une relance de l'emploi à travers un soutien des PME " rentables".

II demeure difficile, pour l'instant, de départager les effets des restructurations en cours sur les groupes sociaux et sur les régions. Même si certains principes semblent se dégager - on dira par exemple que les reconversions industrielles sont possibles dans les milieux où l'emploi n'a pas été accaparé, depuis plusieurs générations, par des grandes entreprises spécialisées ${ }^{11}$ - ceux-ci relèvent davantage de l'observation empi- 
rique que de la théorie. Même si certains phénomènes, comme le changement d'attitude des pouvoirs municipaux à l'égard du développement, traduisent concrètement l'émergence de dynamismes locaux et régionaux, nous connaissons mal leur ampleur et leur portée.

II n'en reste pas moins que sur le terrain les effets de la crise ne passent pas inaperçus. Même si la montée des dynamismes locaux et régionaux - que traduit le projet de consolidation des pouvoirs municipaux émanant des instances municipales elles-mêmes - peut être interprétée comme étant une résultante de la crise, compte tenu de son ampleur, lorsqu'elle dépasse le seuil de l'épiphénomène pour mettre en place les conditions de définition d'une nouvelle réalité sociale, elle contribue à influencer les rapports de force sur la scène politique et génère des effets autonomes.

En ce sens les enjeux locaux sont bel et bien, dans le contexte de crise, au coeur de la redéfinition des rapports État-société. Et audelà de la place ou du rôle que l'on attribue aux collectivités locales s'affrontent des conceptions opposées du développement. Nous pouvons penser ici au débat, à l'intérieur de la " science régionale ", entre les tenants du développement par le haut et ceux du développement par le bas ${ }^{12}$. Ce quii rejoint aussi l'opposition dont parle Yves Barel entre une conception " capitalistique " et une conception " patrimoniale " 13 du développement. Mais, de manière plus pragmatique, nous nous référons aux stratégies concrètes des acteurs socioéconomiques qui, soit continuent de valoriser le développement externe, soit tentent d'impulser un développement alternatif à partir des spécificités locales et de leur capacité d'innovation.

En référence à cette dernière perspective, le pouvoir local s'inscrit à l'intérieur d'une nouvelle pro- blématique du développement qui considère que la dimension économique n'est pas un secteur autonome, évoluant à l'intérieur d'une sphère close mais est, au contraire, en interaction constante avec les dimensions sociales territoriales, culturelles, politiques. Par ailleurs cette problématique met de l'avant des " attitudes innovatrices " tant sur le plan de l'utilisation des technologies nouvelles que sur celui de la formation, de la participation, de la mise en place de multiples réseaux de communication et d'échange, et même sur ceux des services et de l'expertise.

Si cette problématique du développement a d'abord été revendiquée par des organismes communautaires et des mouvements régionaux - soucieux de se soustraire aux exigences imposées par le modèle du développement polarisé -, voilà qu'elle apparaît de plus en plus comme une condition de survie non seulement pour les régions périphériques mais aussi pour la majorité des régions et des collectivités locales. Comme le souligne Walter B. Stöhr, " la dynamique externe engendrée par la croissance globale est devenue manifestement incapable de fonder un développement régional soutenu14 ${ }^{14}$. Ce qui ne signifie pas pour autant qu'il suffise d'évoquer les vertus du micro-développement pour résoudre ce problème!

Le développement local n'est pas possible à n'importe quelles conditions. Les obstacles demeurent nombreux.

L'aliénation créée par un siècle de travail dépendant, de mode de vie contraint, d'intercessions multiples séparant les travailleurs non seulement des fruits de leur travail, mais des moyens de peser sur leur destin, tout cela prépare mal à prendre directement en main la responsabilité de son avenir ${ }^{15}$

John Friedman va même plus loin en soutenant que l'autodéveloppement ne peut être une stratégie viable pour les villes et les régions " périphériques ". Selon lui, seuls les espaces polarisés par les métropoles mondiales, qui occupent une position privilégiée en tant que " centres de contrôle, de production et d'accumulation du capital ${ }^{16}$ ", sont en mesure de se prévaloir d'un développement endogène. En contrepartie John Friedman propose une alternative plus radicale pour les zones de la périphérie, un développement qu'il qualifie d'autodépendant (self-reliant). Situé sur un registre différent du capitalisme mondial, ce développement renvoie avant tout aux dynamismes de la société civile et correspond à " l'émergence d'une pratique sociale radicale" qui se démarque des rationalités de l'État et du grand capital.

Cette pratique, si elle est bien comprise, peut jeter les bases d'une révolution dans l'ordre social pour ces collectivités menacées d'effritement par leurs propres contradictions. Une société autodépendante est ouverte à tous, non hiérarchisée, guidée par la coopération plutôt que par la compétition, l'harmonie avec la nature plutôt que son exploitation, les besoins sociaux plutôt que les désirs personnels insatiables. Cette nouvelle société représente d'ailleurs la meilleure chance de survie pour la race humaine ${ }^{17}$.

Mais le choix en faveur d'un développement écologique et convivial ne résout pas toutes les incertitudes. C'est le moins que l'on puisse dire! Non seulement cette stratégie risque de faire le lit de la dualisation sociale, mais en plus il est difficile d'établir les conditions de sa réussite. En outre il faut bien voir que cette stratégie est formulée au moment même où les collectivités locales sont interpellées par des projets gouvernementaux de décentralisation. 
L'autonomie des collectivités locales: aux seuls enjeux de la décentralisation. Pourtant, cette réforme qui fait parler d'elle au Québec comme ailleurs se situe dans un certain sens au coeur des transformations des modes de gestion du social. Et en tant que telle, elle est traversée d'une perpétuelle ambivalence qui en fait à la fois l'instrument d'une adaptation de l'État aux nouvelles règles du jeu en conjoncture de crise, et un projet porté par certains acteurs sociaux à la conquête des conditions de leur autonomie. Pour comprendre la complexité de cette ambivalence, il convient de dépasser une fois pour toutes une interprétation abordant les enjeux de la décentralisation à travers un schéma binaire, central/local, dans lequel ces termes se trouveraient réifiés. Au Québec notamment, les analyses sont restées longtemps prisonnières d'une opposition tranchée entre un pouvoir local identifié aux élites traditionnelles et un pouvoir central assimilé à une technocratie modernisatrice. Cette vision n'appartient-elle pas à une autre "époque ", celle des années soixante où, dans un contexte d'expansion économique et de développement de l'appareil d'État, les premiers projets de restructuration des institutions locales - dont les projets de fusion municipale et de fermeture de paroisses dans l'Est du Québec - s'inscrivaient dans une problématique de modernisation sociétale dont l'État était l'emblème et l'agent principal, et qui s'exprimaient à travers l'idéologie du rattrapage. Le « local " était alors identifié à la tradition, vu comme un frein au changement, comme un reliquat de modes de fonctionnement désuets susceptibles d'entraver la rationalisation et la mobilisation des ressources requises par la mobilité spatiale d'entreprises. La mise en place d'infrastructures et d'institutions susceptibles de soutenir la modernisation de l'économie, mais aussi l'instauration de mécanismes de justice distributive ne pouvaient qu'émaner du * central ", face à un pouvoir local caractérisé par sa force d'inertie et son conservatisme.

Les projets de restructuration de l'appareil d'État relancés à la fin des années soixante-dix reposent sur de tout autres bases et s'inscrivent dans une conjoncture économique et politique passablement différente. Avant de faire le point sur ces changements de conjoncture et de présenter les réformes et les débats en cours au Québec, il est utile de souligner les différences accusées par la restructuration de l'appareil d'État selon les domaines d'intervention. En effet, alors que le Québec n'a peut-être pas beaucoup d'équivalents en ce qui concerne l'ampleur de la modernisationcentralisation des secteurs de la santé, de l'éducation et de façon générale des affaires sociales, les tentatives de réforme des structures municipales et d'encadrement institutionnel de l'urbanisme et de l'aménagement du territoire ont échoué plus souvent qu'autrement. Alors que dès 1965 on restructure les commissions scolaires, qu'en 1972 on réorganise le système des soins de santé et des services sociaux, il faut attendre 1979 pour que soit enfin adoptée la loi 125 sur l'aménagement et l'urbanisme. Cette loi fait d'une pierre deux coups : elle instaure une certaine régionalisation des structures municipales en créant les municipalités régionales de comté (MRC) (regroupant un ensemble de municipalités rurales et urbaines) et elle exige que les nouvelles instances, les MRC, préparent et adoptent des schémas d'aménagement. Si cette loi ne constitue, selon l'expression de Jacques Léveillée, qu'un " moment de plus dans le processus tortueux de l'aménagement au Québec ${ }^{19}$ ", personne n'est dupe de ses résonnances face à une perspective de décentralisation. C'est ici qu'il faut en effet faire intervenir le contexte plus général qui quence, ce qui se joue au niveau du pouvoir local ne se réduit pas 
a présidé à son adoption. Si les avant-projets de loi sur l'urbanisme et l'aménagement du territoire ont été à l'origine formulés dans une conjoncture d'expansion économique et d'essor d'une politique étatiste, tel n'est plus le cas lorsque dans la deuxième moitié des années soixante-dix paraissent les projets de livre blanc sur la décentralisation et de loi sur l'aménagement et l'urbanisme.

Contexte de crise économique et de crise de l'État-providence certes, mais aussi de modification progressive des rapports entre l'État et les collectivités locales. On serait tenté de dire que l'État a commencé à jouer la carte du local pendant que le pouvoir municipal se muait en acteur " central "! Les tentatives répétées de restructuration des structures municipales ont en effet interpellé les gouvernements locaux et ont, à terme, induit un certain déblocage. Dénonçant fermement ce qui était perçu comme des visées centralisatrices et normalisatrices du gouvernement provincial, les municipalités ont peu à peu cessé d'apparaître comme le bastion par excellence de l'immobilisme et de l'antimodernisme à travers l'Union des municipalités du Québec et I'Union des municipalités régionales de comté, les municipalités rurales et urbaines ont affirmé des points de vue spécifiques et souvent convergents face à une série de dossiers - fiscalité municipale, aménagement, décentralisation... - particulièrement stratégiques en ce qui concerne les enjeux locaux et régionaux. Délaissant progressivement une position définie par leur force d'inertie, elles ont manifesté beaucoup de détermination dans la défense de leur autonomie. C'est qu'en plus d'être interpellées par les projets de réforme de l'État provincial, elles étaient aussi « stimulées » en quelque sorte par une conjoncture économique obligeant de plus en plus les élus locaux à penser davantage en termes de stra- tégies de développement. Tant la conjoncture de crise que l'émergence de mouvements régionaux embryonnaires les incitaient à occuper de manière plus ou moins dynamique un espace politique qu'on leur concédait par défaut. Bien sûr, elles sont loin d'être toutes entrées dans le mouvement avec la même intensité! Les stratégies qu'elles adoptent face aux projets du gouvernement provincial sont souvent très différentes ${ }^{20}$. Enfin, les élus locaux sont loin de constituer, encore aujourd'hui, une frange politique caractérisée par son ouverture et son esprit d'innovation. II n'en reste pas moins qu'ils se sont constitués en interlocuteurs " centraux " pour l'État et que ce dernier a ajusté ses stratégies en conséquence. Dès la fin des années soixante-dix, la revalorisation du pouvoir local est devenue la pierre angulaire du réaménagement des rapports entre l'État et les municipalités. La loi 72 sur la fiscalité municipale octroyant aux municipalités l'exclusivité du champ de l'impôt foncier, la loi 105 sur la démocratie et la rémunération des élus dans les municipalités, témoignent chacune à leur manière du nouveau rôle que l'État veut faire jouer non seulement aux municipalités mais aussi aux citoyens et aux organismes du milieu. Le discours gouvernemental sur la réforme des institutions locales aurait-il, depuis les années soixante, effectué un sérieux virage, délaissant la bannière de la planification globale donc centrale, pour celle, plus légère, du pouvoir local?

Tout n'est pas si simple.

Les réformes législatives évoquées précédemment, et qui semblent aller dans le sens d'une revalorisation du pouvoir local, doivent en effet être replacées dans l'ensemble des mesures et projets de l'État québécois. Tous les segments de l'appareil d'État sont loin d'avoir épousé avec une force égale la philosophie de la décentralisation. Ainsi l'adoption de la loi 125 sur l'amé- nagement du territoire et de l'urbanisme n'a pas empêché la mise en vigueur de lois sectorielles fortes épousant une perspective centralisatrice et ce, dans des domaines névralgiques pour les questions d'aménagement. La loi 90 constitue à cet égard un exemple probant puisqu'elle confie à un organisme provincial la protection du territoire agricole, qui approuve les plans de zonage des municipalités en cette matière.

En fin de compte, les orientations centralisatrices de certaines politiques gouvernementales, les zones d'ombre d'un document comme celui du ministre délégué à l'aménagement et au développement régional - Le choix des Régions - (qui ne crée pas une véritable structure décentralisée en mettant sur pied des tables de concertation qu'il nomme Conseil régional de concertation et d'intervention (CRCl) et dont l'objectif est de permettre aux milieux locaux d'intervenir plus efficacement dans le développement des régions), font douter de la volonté gouvernementale de renouveler les modes de gestion traditionnels. Même si la loi 125 sur l'aménagement du territoire force le gouvernement à faire connaître aux instances locales les projets de ses ministères, elle ne met pas en place à proprement parler une structure décentralisée dérivée d'une volonté politique de redéfinition des rapports de pouvoir et confiant aux instances décentralisées des responsabilités et des pouvoirs réels. Les velléités décentralisatrices resteraient moulées à l'intérieur d'un cadre autonormalisateur en même temps et surtout parce qu'elles sont contrebalancées par une intention ferme de maintenir intacte, sur l'essentiel, la répartition des pouvoirs.

On notera du reste qu'au chapitre de la décentralisation, de nombreux changements ne sont encore que de l'ordre du discours. Certes ce discours fait la part belle au local, 
Revue internationale d'action communautaire $\quad 13 / 53$

L'autonomie des collectivités locales :

entre la crise et le changement

42 aux dynamismes communautaires, pour un peu il évoquerait une perspective autogestionnaire et en tout cas conviviale! Est-il en fin de compte si différent de celui que développent certains groupes et certains mouvements qui ont à coeur de promouvoir des modèles de développement local endogène? S'agit-il alors, de la part de l'État, d'une opération démagogique? S'agit-il au contraire d'un consensus social en train de se cristalliser à partir du contexte de crise et à travers la restructuration de l'appareil d'État?

$\mathrm{Si}$, à condition d'adopter une position optimiste, on peut repérer les premiers signes d'un tel consensus, ce dernier est en tout cas loin d'être acquis en ce qui concerne le territoire considéré de part et d'autre comme le lieu privilégié du pouvoir local. Pendant que les municipalités réaffirment la coïncidence de ce lieu avec le territoire municipal, le gouvernement provincial quant à lui défend dans ses projets de décentralisation, une conception du local identifiant ce dernier à de nouveaux espaces politicoadministratifs qu'il tente de créer de toutes pièces à l'échelon supralocal. En outre, cet échelon est à son tour scindé en deux : le territoire des MRC et celui des $\mathrm{CRCl}$, le premier étant celui de l'aménagement du territoire, le second celui de la concertation relative aux orientations du développement socio- économique. Cette différenciation balkanise en quelque sorte ce qui a trait, de façon forcément restrictive, à l'aménagement du territoire et ce qui a trait au développement socio-économique ${ }^{21}$. L'idée des $\mathrm{CRCl}$ et d'ailleurs l'ensemble du document Le choix des Régions ont soulevé auprès des élus locaux un tollé de protestations : ces tables de concertation où seraient représentés la plupart des intervenants du " milieu " consacreraient la relégation de l'élu local au rôle d'intervenant parmi les autres face au gouvernement provincial ${ }^{22}$.

Par delà les récriminations suscitées à l'égard du projet des $\mathrm{CRCI}$, il faut se rappeler que ce n'est pas la première fois qu'un État crée un espace politico-administratif sous couvert de démocratie : la notion de planification régionale n'est-elle pas née à la fin des années trente à l'occasion de l'aménagement de la vallée du Tennessee par le gouvernement fédéral américain ${ }^{23}$. $\mathrm{Ne}$ serait-ce pas pour l'État une manière de contrôler les conditions de la concertation qu'il souhaite par ailleurs?

Chose certaine, les débats sur la décentralisation sont loin d'être clos. Car au Québec la redéfinition du partage des compétences respectives de l'État et des collectivités locales, et la remise en question du modèle de l'État-providence, n'ont pas encore été discutées à fond. Les réformes engagées sont somme toute assez timides et une bonne partie des changements envisagés sont encore de l'ordre du discours, on l'a dit.

\section{Les mouvements sociaux entre la politique locale et le corporatisme}

La gestion du social fait appel, en ce moment, à une nouvelle problématique de l'intervention de l'État qui suppose une redéfinition des rapports entre l'État et la société civile. Dans ce contexte les restructurations économiques et les réformes institutionnelles prennent un sens particulier. La médiation politique des intérêts sociaux se trouve caractérisée par une crise de la représentation politique à travers laquelle s'exprime la montée d'un néo-corporatisme.

Ce sont là des éléments indispensables à prendre en compte pour définir la manière dont les acteurs sociaux auront à se situer sur la scène locale face aux transformations des modes de gestion traditionnels de l'État. Un certain nombre de forces sociales sont déjà en train de redéfinir leurs stratégies à ce niveau. Le regain de vigueur des chambres de commerce, notamment à Montréal, les sollicitations dont certains groupes d'intérêts sont l'objet de la part des gouvernements tant locaux que provinciaux, les formules de concertation que ces mêmes gouvernements favorisent à l'instar des $\mathrm{CRCl}$, constituent autant d'indices de l'affirmation d'un néo-corporatisme aux dépens des canaux institués de représentation politique.

Ce modèle corporatiste n'est certes pas nouveau. À Montréal, il a dominé la vie politique municipale pendant près de 20 ans, un tiers du Conseil municipal étant directement désigné par les associations représentant le monde des affaires, le monde ouvrier, celui de l'éducation, les milieux réformistes et les propriétaires. La doctrine corporatiste était particulièrement répandue après la Seconde Guerre, grâce notamment aux bons soins de l'Église qui y voyait un moyen de canaliser de manière non conflictuelle les rapports entre forces 
sociales et ce, en dehors de l'État. Aujourd'hui, il s'agit d'un néocorporatisme qui s'affiche moins en tant que tel mais qui court-circuite tout autant que son " prédécesseur " la dynamique de la représentation politique.

Si pour reprendre les termes de Bernard Eme, il faut dénoncer la vision mécaniste qui consiste à confondre l'accroissement " des responsabilités des élus locaux et le rapprochement du pouvoir des citoyens pour leur en faciliter l'accès et en démocratiser l'exercice ${ }^{24}$ ", il faut peut-être et surtout évaluer les risques que fait peser ce néo-corporatisme sur le jeu démocratique. Au moment où le local semble pouvoir (re)devenir le lieu d'une politisation des débats sur la gestion sociale à travers une réarticulation des enjeux du développement économique et ceux de la redistribution sociale, on favorise des formes institutionnelles et organisationnelles de négociation entre partenaires sociaux, qui privilégient les groupes d'intérêts traditionnellement bien organisés. Mais ce faisant, tout un éventail de forces sociales se trouvent marginalisées, toute une série d'enjeux conflictuels se trouvent évacués. C'est bien ce qui ressort, par exemple, des sommets économiques régionaux impulsés par le gouvernement provincial, sommets qui n'en ont pas moins, par ailleurs, permis l'expression de certains dynamismes locaux.

$\mathrm{Ce}$ néo-corporatisme qui exprime et entretient une revitalisation de certains secteurs de la société civile masque la marginalisation de catégories sociales sans cesse croissantes que la crise économique a engendrées, même parmi les fractions instruites des nouvelles classes moyennes. II constitue, dans ce sens, l'équivalent au plan politique, de la dualisation sociale. Mais il pénalise aussi les groupes constitués d'acteurs en position sociale précaire ou qui interviennent à propos d'enjeux qui ne sont pas considérés comme prioritaires et, de façon plus générale, il contribue à l'affaiblissement des formes de médiation politique des intérêts sociaux sur lesquelles repose la démocratie représentative.

Pour bien saisir la portée de ce néo-corporatisme il est important d'ouvrir une parenthèse afin de considérer la nature des transformations qui lui ont permis de s'incruster. Le blocage des mécanismes de croissance a provoqué d'une part l'émergence de nouveaux modèles de développement économique et, d'autre part, la définition de nouveaux modèles de développement social ou de gestion du social. D'un côté l'on comprend que la croissance n'est pas un phénomène induit par des forces exogènes incontrôlables - même si l'on a encore parfois tendance à penser que c'est toujours le cas pour la décroissance - et que finalement il demeure possible, à certaines conditions, d'infléchir le développement. De l'autre l'on semble admettre de plus en plus que la redistribution doit s'effectuer de manière différente.

Non seulement sont remis en cause - et encore là à partir de points de vue divers - les valeurs égalitaires qui fondaient l'action redistributrice de l'État-providence, mais également l'on considère qu'il n'est plus possible d'élaborer des politiques sociales qui ne tiennent pas compte de l'évolution du système de production. $\mathrm{Si}$, dans les années soixante et soixante-dix, les critiques de gauche à l'endroit de la gestion étatique du social s'articulaient à des valeurs de justice sociale - prenant implicitement pour acquis que la croissance allait se poursuivre ad infinitum - la transformation des modèles de développement sape tout d'un coup leur fondement. II n'est plus possible de penser comme si le défi premier était de réduire les inégalités sociales - entre autres en établissant une égalité des chances
- sans remettre en cause, dans les faits, les rapports de production, l'organisation du travail, l'accès à l'emploi. À la lumière de la crise, il apparaît plus clairement que l'Étatprovidence a davantage cherché à compenser les "dégâts du progrès " qu'il n'a visé à transformer la structure des inégalités sociales. Or voilà que les mesures de compensation - et de redistribution qui avaient dans les faits (jusqu'à un certain point) permis d'atténuer les effets des inégalités sociales, à tout le moins pour une partie des classes populaires et des classes moyennes, sont progressivement diminuées, risquant même, dans certains cas, de disparaître. D'autres avenues doivent donc être explorées quant à la manière de concevoir les politiques sociales. S'il est indispensable, comme le souligne Christine Buci-Gluckmann ${ }^{25}$, d'élaborer une "nouvelle philosophie de la justice sociale ", celleci ne sera possible qu'à la condition d'inscrire les principes de la redistribution dans les limites d'un modèle de développement sur lequel les collectivités locales auront prise.

En ce sens les enjeux du développement, dans leur articulation au local, prennent une configuration particulière qui interpelle tout autant les nouvelles classes moyennes, les exclus du système de production que la classe politique ellemême. S'il n'existe pas de modèle de développement idéal, au risque d'aggraver les inégalités existantes, on ne pourra plus gérer le développement économique sans introduire, déjà à ce niveau, la question de l'action collective et de la place des diverses catégories d'acteurs. II semble qu'il sera de plus en plus difficile de gérer après coup comme on le faisait dans le passé - les méfaits de la croissance.

Ces remarques nous conduisent à considérer différemment le rééquilibrage des forces sociales dans la mesure où les changements dont 
44 il a été question sont porteurs de différenciations quant à la manière dont les groupes se situent sur la scène locale.

Par exemple, à l'intérieur de cet ensemble composite qu'on appelle les nouvelles classes moyennes, l'embourgeoisement des uns n'a d'égale que la marginalisation des autres. Les premiers, soucieux de consolider leurs acquis, affirment leur présence sur la scène locale autour d'enjeux néo-conservationnistes (protection des milieux naturels et bâtis, qualité de la vie) et se rapprochent bien malgré eux, des positions d'une élite traditionnelle qui, ces dernières années, a effectué un sérieux rattrapage idéologique. Les seconds se caractérisent par une insécurité économique qui, au mieux, les fait basculer vers une contre-culture tendant à évacuer le politique; ce qui contraste avec l'investissement des premiers dans la démocratie locale. Nous faisons même l'hypothèse que cette dernière est pour les nouvelles classes moyennes embourgeoisées ou en voie de l'être, un " moyen de se constituer en force sociale" comme le fut jadis la démocratie locale en France au bénéfice de la petite bourgeoisie ${ }^{26}$.

Du point de vue des mouvements sociaux, la question est encore plus complexe car, en plus de constater le recul des forces populaires depuis les années soixante, il faut surtout souligner l'ex- trême diversité des enjeux, des modes d'organisation et des composantes sociales de ce que l'on appelle aujourd'hui les nouveaux mouvements sociaux. Cette constellation est loin d'être stabilisée et la redéfinition des rapports entre État et société civile ne facilitera pas la cristallisation de ces mouvements épars dans des formes qui permettraient la mise sur pied de coalitions sur la scène politique locale.

Pour l'instant, les différentes composantes des nouveaux mouvements sociaux ne semblent pas avoir pris au sérieux les enjeux du pouvoir local. Soit que leurs revendications et leurs interventions se situent en deça. C'est le cas notamment de nombreux mouvements alternatifs - on peut penser à certaines coopératives, aux mouvements d'autoconstruction, d'autosanté, etc., pour lesquels le politique constitue un lieu piégé. Soit que leur action porte au-delà du local à l'intention de la classe politique qui manoeuvre dans les appareils d'État centralisés. On peut penser alors aux garderies dont la stratégie consiste à élaborer un rapport de force à l'État pour qu'il adopte une politique nationale. Mais il y a aussi l'exemple de certains groupes écologistes. Au Québec, certaines fractions du mouvement écologiste s'imposent en interlocuteur privilégié face à l'État dans une forme de débat - d'expert à expert - qui renforce la logique étatiste. Michel Marié ${ }^{27}$ fait un constat similaire pour le Sud de la France : l'analyse des acteurs privilégiés du débat environnemental révèle que la "société locale " tend à être considérée avec suspicion même par les groupes écologistes.

S'agit-il là de stratégies cherchant à éviter, lucidement, les embûches du pouvoir local, ou s'agit-il de stratégies basées sur l'idée que l'État-providence existe toujours?

La question du pouvoir local ne nous semble, pourtant pas, “ contournable " à moyen terme. Nous faisons même l'hypothèse qu'elle peut être porteuse à l'avenir d'une repolitisation des enjeux du développement à travers une réarticulation des dimensions économique et sociale, et que les mouvements sociaux ne peuvent pas se permettre, si l'on ose dire, de ne pas être partie prenante.

Annick Germain et Pierre Hamel Institut d'urbanisme Université de Montréal 


\section{NOTES}

1 Yves Barel, “ Modernité, code, territoire ", Les Annales de la recherche urbaine, $n^{\text {os }} 10$ et 11, printemps 1981, p. 7.

2 Voir à ce propos Jean-Claude Thibodeau, Pierre-André Julien, “ Les PME manufacturières dans la tourmente économique de 1975 à 1982 ; un premier bilan dans quatre régions du Québec ", Montréal, I.N.R.S.-Urbanisation, 20 p.

3 Pour une revue de ces analyses voir Clyde Weaver, "Development Theory and the Regional Question : A Critique of Spatial Planning and its Detractors", W. B. Stöhr, D. R. Fraser Taylor, Development from above or below, New York, John Wiley and Sons, 1981, p. 73-105.

4 Voir Philippe Aydalot, “ À la recherche des nouveaux dynamismes spatiaux", P. Aydalot, Crise et espace, Paris, Economica, 1984, p. 38 et suivantes.

5 Philippe Aydalot, op. cit., p. 41.

6 Philippe Aydalot, op. cit., p. 54

7 Philippe Aydalot, op. cit., p. 56. Mais voir aussi le numéro 1, 1983, de la Revue d'économie régionale et urbaine, réalisé par les chercheurs du Centre Économie, Espace, Environnement (Université de Paris 1, Panthéon, Sorbonne).

8 Voir en particulier, Gouvernement du Qué bec, ministre François Gendron, Le choix des Régions (Document de consultation sur le choix des régions), Québec, 1983 , $132 \mathrm{p}$.

9 Comme l'écrivent Pierre Maclouf et JeanLouis Sarbib, “ Le redéploiement vise surtout à la création de quelques pôles industriels lourds, liés à des groupes potentiellement compétitifs au niveau mondial ". "Dualisation spatiale et dualisation sociale de la force de travail en période de crise : deux scénarios possibles", Revue d'économie régionale et urbaine, $\mathrm{n}^{\circ} 1,1983$, p. 177.

10 François Dalpert, « De la croissance duale à l'expérimentation sociale ", Revue internationale d'action communautaire, 1980 , $\mathrm{n}^{\circ} 3$, p. 160.

11 Voir, entre autres, à ce sujet les remarques de Philippe Aydalot, “Crise économique, crise de l'espace, crise de la pensée spatiale ", B. Planque, Le développement décentralisé, Litec, collection GRAL, $n^{\circ} 16,1983$, p. 97. Comme il le soulignait également ailleurs : “On peut observer, sans doute avec surprise, que les initiatives les plus prometteuses apparaissent depuis dix ans dans les milieux relativement peu marqués par l'industrie : en Italie, au Portugal, en Tunisie même, les exemples abondent de créations multiples, sur la base de techniques simples
[...] ". Philippe Aydalot, "Réalités et illusions de l'économie locale ", Autrement, $n^{\circ} 47$, février 1983, p. 210.

12 Voir Walter B. Stöhr, “ Development from below: the bottom-up and peripheryinward development paradigm ", W. B. Stöhr, D. R. Fraser Taylor, op. cit., p. 39-71.

13 Yves Barel, op. cit., p. 17 et suivantes.

14 Walter B. Stöhr, "La crise économique demande-t-elle de nouvelles stratégies de développement régional ", P. Aydalot, op. cit., p. 190.

15 Philippe Aydalot, " Réalités et illusions de l'économie locale ", op. cit., p. 210.

16 John Friedman, " Auto-développement ou auto-dépendance ", M. Boisvert, P. Hamel (éds), Redéploiement industriel et planification régionale, Montréal, Faculté de l'aménagement, 1983, p. 298.

17 John Friedman, op. cit., p. 297.

18 Voir l'étude de Gérard Divay et Jacques Léveillée avec la collaboration de Bernard McCaan, La réforme municipale de l'État québécois (1960-1979), Montréal, I.N.R.S. Urbanisation, 1981

19 Jacques Léveillée, "Introduction ", J. Léveillée (sous la direction de), L'Aménagement du territoire au Québec. Du rêve au compromis, Montréal, Nouvelle Optique, 1982.

20 Voir Rita Bissonnette, "L'Union des municipalités régionales de comté du Québec : la stratégie des petites patries ", A. Germain, P. Hamel (éds), Aménagement et pouvoir local, Montréal, ACFAS, 1985.

21 Ce genre de distinction avait déjà été esquissé il y a quelques années par l'Office de planification et de développement du Québec qui, constatant l'emprise totale des ministères sectoriels sur le champ du développement socio-économique en matière d'intervention gouvernementale, s'était stratégiquement replié sur l'aménagement de “ l'espace " pour définir sa vocation et ses prérogatives.

22 " Le CRCl serait composé, selon des modalités qui tiennent compte des caractéristiques de chacune des régions, d'une vingtaine de représentants élus, mandatés par les milieux suivants :

- le monde municipal (préfets et maires) - le milieu des affaires (groupements d'entreprises, chambres de commerce et coopératives)

- le milieu des travailleurs, des producteurs agricoles et forestiers, des syndicats - les institutions d'enseignement (commissions scolaires, CÉGEP, universités) et le réseau de la santé et des services sociaux (CRSSS)

- les conseils consultatifs et les diverses associations de la région (Conseil régional de la culture, Conseil régional des loisirs, Association touristique régio- nale, etc.)"

Le ministre Francois Gendron, Le choix des Régions, documentation de Consultation sur le développement des régions, 1983, p. $98-99$.

23 Philippe Selznick, TVA and the Grass Roots Democracy, New York, Harper Torchbooks, 1966.

24 Bernard Eme, "Le local verrouillé ", Autogestions, $n^{\circ} 15,1984$.

25 Christine Buci-Gluckmann, "Pour une nouvelle philosophie de la justice sociale ", Changement social en France et en Europe ?, Paris, Syros, 1982.

26 Jean-Yves Nevers, " Hégémonie et démocratie locale ", R. Ledrut, Le pouvoir local, Paris, Anthropos, 1979.

27 Michel Marié, Un territoire sans nom. Pour une approche des sociétés locales, Paris Librairie des méridiens, 1982. 